\title{
Abstracts from the European Respiratory Society Annual Conference 2017 on respiratory critical care
}

\author{
Murali Shyamsundar ${ }^{1}$, Lieuwe D. J. Bos ${ }^{2}$, Raffaele Scala ${ }^{3}$ \\ ${ }^{1}$ School of Medicine, Dentistry and Biomedical Sciences, Centre for Experimental Medicine, Institute for Health Sciences, Belfast, UK; ${ }^{2}$ Department \\ of Intensive Care, Emergency Medicine, Infectious Diseases, Respiratory Medicine, University of Amsterdam, Amsterdam, Netherlands; ${ }^{3}$ Respiratory \\ Division with Pulmonary Intensive Care Unit, S. Donato Hospital, AUSL 8 Arr., Arrezzo, Italy \\ Correspondence to: Lieuwe D. J. Bos. Department of Intensive Care, Emergency Medicine, Infectious Diseases, Respiratory Medicine, University of \\ Amsterdam, Amsterdam, Netherlands. Email: lieuwe.bos@gmail.com.
}

Submitted Aug 11, 2017. Accepted for publication Sep 09, 2017.

doi: $10.21037 /$ jtd.2017.11.46

View this article at: http://dx.doi.org/10.21037/jtd.2017.11.46

The recently concluded European Respiratory Society (ERS) Annual International Congress 2017 provided the international audience results from a range of research topics. The Congress provided a platform for diverse areas covering studies from bench to bedside research reflecting the broad appeal of ERS. Exciting research was presented from animal experiments, observational studies and interventional trials. In this article, we discuss what are in our opinion the top four abstracts presented at the Congress which will be of interest to our readers. They explore varied novel concepts dealing with technology during mechanical ventilation, pharmacotherapy targets and non-invasive respiratory support tools to improve the safety and effectiveness in the management of critically ill patients with respiratory conditions.

\section{Renin-Angiotensin system (RAS)—expanding role in the critically ill}

The RAS is of increasing interest for critical care. RAS is an essential physiological responder to shock including septic shock. In addition to haemodynamic effects, angiotensin II (Ang 2) is also implicated in various biological processes such as inflammation, apoptosis and cellular growth. The activation of RAS produces widespread physiologic changes, which are in many instances of opposite effects (i.e., detrimental and protective). This depends on the balance of activation between angiotensin receptor type 1 (AT-1) and type 2 (AT-2) (1). This interesting and opposing effect of RAS activation is hypothesized to be secondary to angiotensin converting enzyme (ACE)/ACE2 balance resulting in varying ratios in Ang II/Ang1-7. This imbalance leads to Ang II activation of AT1 receptor and is implicated in the development of acute respiratory distress syndrome (ARDS) while many of these detrimental effects are ameliorated by Ang II metabolite, Ang1-7 (2). The heptapeptide Ang1-7 acts via G protein-coupled receptor Mas to express their protective functions.

To this growing body of evidence for the protective role of Ang1-7 (3) has added protection against ventilator induced diaphragmatic dysfunction (VIDD). Diaphragmatic dysfunction due to muscle atrophy is known to be an early phenomenon in patients undergoing mechanical ventilation. While different ventilator modes are proposed to reduce this, the pathomechanisms behind this deleterious effect of ventilation are still unclear. Using an elegant series of experiments in a rat model, Zambelli et al. have demonstrated the protective effects of Ang1-7. Rats were ventilated for 8 hours to mimic prolonged controlled mechanical ventilation and were randomized between receiving an infusion of Ang1-7 or placebo. While the ex-vivo diaphragmatic contractility did not differ between the two groups of animals, there was significant increase in muscle cross sectional area and a reduction in myogenin mRNA levels, which were restored after administration of Mas receptor blockade. This body of bench side research has the potential to translate to bedside to prevent VIDD in mechanically ventilated patients. Of course, other various questions, such as the limitations of a relatively short duration of ventilation in an animal model, the modality 
and setting of ventilation, timing of therapy, effects of vasodilatation and anti-inflammatory effects need to be addressed in critically ill septic patients and other patients at higher risk of developing VIDD.

\section{Relevance of high flow nasal cannula oxygen (HFNO) in chronic respiratory conditions}

HFNO is a game changing therapy gaining increasing popularity in acute care of hypoxemic patients. HFNO delivers oxygen up to $100 \%$ with flows of up to $60 \mathrm{~L} / \mathrm{min}$. This has paved the way for its recommendation in various acute scenarios such as acute type 1 respiratory failure, difficult intubation, high risk of extubation failure, endoscopies and in peri-operative patients to prevent respiratory failure, as well end-stage respiratory diseases. The mechanism of action of HFNO is multifactorial and includes provision of low levels of positive end-expiratory pressure (PEEP), adequate humidification, deliver of high inspiratory flow rates to meet patients' ventilatory demands and reduction of anatomical dead space with $\mathrm{CO}_{2}$ washout. The end result is improved secretion clearance, increase oxygenation, prevention of atelectasis as well as augmented patient comfort. There are benefits of HFNO when compared to conventional oxygen therapy in reducing endotracheal intubation in patients with acute hypoxaemic respiratory failure but there is no proven benefit on mortality (4).

While the role for HFNO in acute hypercapnic respiratory failure is still uncertain (5), Longhini et al. (6) have explored the benefits of HFNO in patients with stable hypercapnic respiratory failure established on long-term oxygen therapy (LTOT). In this multicenter randomized cross over trial of 29 patients, HFNO when compared to LTOT led to a significant improvement in $\mathrm{PaCO}_{2}$, $\mathrm{pH}$ and $\mathrm{PTcCO}_{2}$. There was an associated improvement in subjective symptoms as measured by the St. George's respiratory questionnaire. Interestingly, the biochemical and subjective improvements did not translate to improved exercise or functional capacity. While this is disappointing, we need to understand and explain why this may be the case. We could hypothesize that HFNO on top of LTOT may not have an impact on oxygen delivery which may have led to the lack improvement in physical function. There may well be other beneficial effects such as improved mucous clearance and reduced metabolic demand in these patients. While there may be benefits, a successful translation to routine clinical practice does not impinge on just efficacy but also the ease of delivery. HFNO is still a cumbersome device and may affect mobility which could reduce patient compliance and even participation in physical rehabilitation.

These benefits need to be investigated in longer term studies with adequate sample size. This may provide another rationale for the use of HFNO in addition to LTOT in patients with stable hypercapnic respiratory failure.

\section{Utility of electrical impedance tomography (EIT) during ventilatory weaning}

EIT is a technology which has evolved over the last 30 years and is gaining credence in mechanical ventilation. The principle of EIT is the detection of electrical conductivity of tissue to small alternating currents applied using a series of electrodes placed on the thoracic cage. The main purpose of EIT is to support protective lung ventilation by identification of regional differences in ventilation to prevent ventilator induced lung injury (VILI). The role of EIT has been investigated as a supportive tool to study regional variations during recruitment maneuvers, PEEP titration and tidal volume adjustment. The predominant situation for EIT use is around the early phase of ventilation and in patients with acute respiratory distress syndrome (7).

Dreher $e t a l$. have extended the scope of EIT applications to the end of a patient's mechanical ventilation (i.e., weaning) (8). Extubation is preceded by various steps aiming to explore the capability of the patient to successfully transition to unsupported breathing and this is tested through spontaneous breathing trials and $\mathrm{T}$ piece trials. While there is no advantage to any specific method to assess readiness for extubation, the reason for failure is commonly due to ongoing disease process, de-recruitment and cardiac decompensation. In their study, Dreher et al. focused on the ability of EIT to detect de-recruitment during $\mathrm{T}$ piece trials. In a small study of 31 patients undergoing prolonged weaning, they measured Global inhomogeneity index (GI) and $\mathrm{PaO}_{2}$ at three different timepoints. Before initiation of $\mathrm{T}$ piece trial, during and after reinstitution of pressure support ventilation (PSV). They showed a significant increase in GI suggestive of de-recruitment associated with a reduction in $\mathrm{PaO}_{2}$ during the T-piece trial, which disappeared after institution of PSV. Unfortunately, the phenomenon of de-recruitment per se is not a predictor of extubation failure. Further researches should be focused to see if extubation failure is predicted by increasing GI. This may identify a cohort of high-risk patients who may benefit from elective prophylactic use of high flow nasal oxygen 
or non-invasive ventilation post extubation to reduce re-intubation rates.

\section{Mandatory to assisted ventilation in ARDS- providing just the right amount of assistance}

A significant body of work over the last two decades has demonstrated clear evidence of benefit from lung protective ventilation (LPV) for patients with ARDS (9). One of the key tenets of protective lung ventilation is low tidal volume (TV) ventilation where TV of $\sim 6 \mathrm{~mL} / \mathrm{kg}$ predicted body weight (PBW) when compared to conventional ventilation significantly reduced mortality (10). Indeed, this benefit might even extend to patients without ARDS, likely secondary to reduction of biotrauma and development of VILI (11). One of the main challenges facing an intensive care physician is the maintenance of low TV ventilation after moving to one of the assisted modes of ventilation.

In a bench study, Perinel Ragey et al. (12) investigated the impact of different assist modes of ventilation in terms of risk of developing VILI. They investigated the size of TV as well as variability in assisted modes, at two breathing frequencies and effort and with mild, moderate or severe ARDS using the ASL 5,000 lung model. Thirteen different modes of ventilation divided in three categories [volume controlled with mandatory minute ventilation, pressure controlled ventilation (PCV) and pressure support ventilation (PSV)] were tested. TVs were compared using the median (first-third quartiles) with variability in TV derived from the co-efficient of variation.

Their results suggest that the risk of $\mathrm{TV}>6 \mathrm{~mL} / \mathrm{kg}$ $\mathrm{PBW}$ is significantly reduced with airway pressure release ventilation (APRV) and pressure support ventilation with guaranteed volume mode while APRV also led to significant TV variability. Despite their interesting findings, this study by Perinel Ragey et al. is unlikely to conclude the neverending debate of the safest assist mode of ventilation. The observations from the simulator should be transferred to the bedside with caution as patients' respiratory physiology can vary significantly over time. The timing of change from mandatory to assist mode will also need to be carefully considered. As a matter of the fact, an early shift to assist mode with the resultant loss of control over LPV may be of greater harm in terms of VILI development to the patient rather than the type of assist mode of ventilation with some loss of TV control. In practice, irrespective of the mode used, we should make a genuine attempt to keep the patient within the "safe zone" of ventilation. This may require novel methods of continuous monitoring of patient physiology and ventilator parameters to prevent VILI.

\section{Acknowledgements}

None.

\section{Footnote}

Conflicts of Interest: The authors have no conflicts of interest to declare.

\section{References}

1. Corrêa TD, Takala J, Jakob SM. Angiotensin II in septic shock. Critical Care 2015;19:98.

2. Santos RA. Angiotensin-(1-7). Hypertension 2014;63:1138-47.

3. Zambelli V, Rizzi L, Zucca L, et al. Angiotensin-(1-7) effects in a rat model of ventilator-induced diaphragmatic dysfunction (VIDD). Eur Respir J 2017;50:OA300.

4. Ni YN, Luo J, Yu H, et al. Can High-flow Nasal Cannula Reduce the Rate of Endotracheal Intubation in Adult Patients With Acute Respiratory Failure Compared With Conventional Oxygen Therapy and Noninvasive Positive Pressure Ventilation?: A Systematic Review and Metaanalysis. Chest 2017;151:764-75.

5. Roca O, Hernández G, Díaz-Lobato S, et al. Current evidence for the effectiveness of heated and humidified high flow nasal cannula supportive therapy in adult patients with respiratory failure. Critical Care 2016;20:109.

6. Longhini F, Pisani L, Lungu R, et al. High-flow oxygen therapy in hypercapnic patients recovering from an episode of acute-on-chronic respiratory failure. Eur Respir J 2017;50:PA1862.

7. Frerichs I, Amato MB, van Kaam AH, et al. Chest electrical impedance tomography examination, data analysis, terminology, clinical use and recommendations: consensus statement of the TRanslational EIT developmeNt stuDy group. Thorax 2017;72:83-93.

8. Dreher M, Cornelissen C, Bickenbach J, et al. Electrical Impedance Tomography for the assessment of spontaneous breathing trials in patients with prolonged weaning. Eur Respir J 2017;50:OA302.

9. Amato MB, Meade MO, Slutsky AS, et al. Driving pressure and survival in the acute respiratory distress syndrome. $\mathrm{N}$ Engl J Med 2015;372:747-55.

10. Acute Respiratory Distress Syndrome Network, Brower 
RG, Matthay MA, et al. Ventilation with lower tidal volumes as compared with traditional tidal volumes for acute lung injury and the acute respiratory distress syndrome. N Engl J Med 2000;342:1301-8.

11. Determann RM, Royakkers A, Wolthuis EK, et al. Ventilation with lower tidal volumes as compared with

Cite this article as: Shyamsundar M, Bos LD, Scala R. Abstracts from the European Respiratory Society Annual Conference 2017 on respiratory critical care. J Thorac Dis 2017;9(Suppl 16):S1537-S1540. doi: 10.21037/jtd.2017.11.46 conventional tidal volumes for patients without acute lung injury: a preventive randomized controlled trial. Crit Care 2010;14:R1.

12. Perinel Ragey S, Baboi L, Guerin C. Variability of tidal volume in assisted mechanical ventilation in ARDS: a bench study. Eur Respir J 2017;50:OA303. 